\title{
An Application of Generalized Bessel Functions on Certain Subclasses of Analytic Functions
}

\author{
G. Murugusundaramoorthy*, T. Janani \\ School of Advanced Sciences, VIT University Vellore - 632014, Tamilnadu, India \\ *Corresponding author: gmsmoorthy@yahoo.com \\ Received November 02, 2014; Revised December 22, 2014; Accepted January 13, 2015

\begin{abstract}
The purpose of the present paper is to investigate some characterization for generalized Bessel functions of first kind to be in the new subclasses of $\beta$ uniformly starlike and $\beta$ uniformly convex functions of order $\alpha$. Further we point out consequences of our main results.
\end{abstract}

Keywords: univalent, starlike, convex, uniformly starlike functions, uniformly convex functions, Bessel functions

Cite This Article: G. Murugusundaramoorthy, and T. Janani, "An Application of Generalized Bessel Functions on Certain Subclasses of Analytic Functions." Turkish Journal of Analysis and Number Theory, vol. 3, no. 1 (2015): 1-6. doi: 10.12691/tjant-3-1-1.

\section{Introduction}

Let $\mathcal{A}$ be the class of analytic functions of the form

$$
f(z)=z+\sum_{n=2}^{\infty} a_{n} z^{n} \quad z \in \mathbb{U} .
$$

As usual, we denote by $\mathcal{S}$ the subclass of $\mathcal{A}$ consisting of functions which are normalized by $f(0)=0=f^{\prime}(0)-1$ and also univalent in $\mathbb{U}$ Denote by $\mathcal{T}$ the subclass of $\mathcal{A}$ consisting of functions whose nonzero coefficients from second on, is given by

$$
f(z)=z-\sum_{n=2}^{\infty} a_{n} z^{n}, \quad a_{n} \geq 0
$$

For functions $f \in \mathcal{A}$ given by (1) and $g \in \mathcal{A}$ given by $g(z)=z+\sum_{n=2}^{\infty} b_{n} z^{n}$, we define the Hadamard product (or convolution) of $\mathrm{f}$ and $\mathrm{g}$ by

$$
\left(f^{*} g\right)(z)=z+\sum_{n=2}^{\infty} a_{n} b_{n} z^{n_{\varkappa}}, \quad z \in \mathbb{U} .
$$

A function $f \in \mathcal{A}$ is said to be starlike of order $\alpha(0 \leq \alpha<1)$, if and only if

$$
\mathfrak{R}\left(\frac{z f^{\prime}(z)}{f(z)}\right)>\alpha \quad(z \in \mathbb{U}) .
$$

This function class is denoted by $\mathcal{S}^{*}(\alpha)$. We also write $\mathcal{S}^{*}(0)=: \mathcal{S}^{*}$ where $\mathcal{S}^{*}$ denotes the class of functions $f \in \mathcal{A}$ such that $f(\mathbb{U})$ is starlike with respect to the origin. A function $f \in \mathcal{A}$ is said to be convex of order $\alpha(0 \leq \alpha<1)$ if and only if

$$
\mathfrak{R}\left(1+\frac{z f^{\prime}(z)}{f^{\prime}(z)}\right)>\alpha \quad(z \in \mathbb{U}) .
$$

This class is denoted by $\mathcal{K}(\alpha)$ Further, $\mathcal{K}=\mathcal{K}(0)$, the well-known standard class of convex functions. It is an established fact that

$$
f \in \mathcal{K}(\alpha) \Leftrightarrow z f^{\prime} \in \mathcal{S}^{*}(\alpha) .
$$

Let $\mathcal{T}^{*}(\alpha)$ and $\mathcal{C}(\alpha)$ are the class of starlike and convex functions of order $\alpha(0 \leq \alpha<1)$, introduced and studied by Silverman [21].

The class $\beta-\mathcal{U C V}$ was introduced by Kanas and Wi'sniowska [12], where its geometric definition and connections with the conic domains were considered. The class $\beta-\mathcal{U C V}$ was defined pure geometrically as a subclass of univalent functions, that map each circular arc contained in the unit disk $\mathbb{U}$ with a center $\xi,|\xi| \leq \beta(0 \leq \beta<1)$, onto a convex arc. The notion of $\beta$ - uniformly convex function is a natural extension of the classical convexity. Observe that, if $\beta=0$ then the center $\xi$ is the origin and the class $\beta-\mathcal{U C V}$ reduces to the class of convex univalent functions $\mathcal{K}$. Moreover for $\beta=1$ corresponds to the class of uniformly convex functions $\mathcal{U C V}$ introduced by Goodman [10,11], and studied extensively by Rønning $[19,20]$. The class $\beta-\mathcal{S}_{P}$ is related to the class $\beta-\mathcal{U C V}$ by means of the wellknown Alexander equivalence between the usual classes of convex $\mathcal{K}$ and starlike $\mathcal{S}^{*}$ functions. Further the analytic criterion for functions in these classes are given as below(also see $[6,19,20,24])$.

For $-1<\alpha \leq 1$ and $\beta \geq 0$ a function $f \in \mathcal{A}$ is said to be in the class 
(i) $\beta$ - uniformly starlike functions of order $\alpha$ is denoted by $\mathcal{S}_{P}(\alpha, \beta)$ if it satisfies the condition

$$
\mathfrak{R}\left(\frac{z f^{\prime}(z)}{f(z)}-\alpha\right)>\beta\left|\frac{z f^{\prime}(z)}{f(z)}-1\right|, \quad z \in \mathbb{U}
$$

and

(ii) $\beta$ - uniformly convex functions of order $\alpha$ denoted by $\mathcal{U C V}(\alpha, \beta)$, if it satisfies the condition

$$
\mathfrak{R}\left(1+\frac{z f^{\prime \prime}(z)}{f^{\prime}(z)}-\alpha\right)>\beta\left|\frac{z f^{\prime \prime}(z)}{f^{\prime}(z)}\right|, \quad z \in \mathbb{U} .
$$

Indeed it follows from (4) and (5) that

$$
f \in \mathcal{U C V}(\alpha, \beta) \Leftrightarrow z f^{\prime} \in \mathcal{S}_{P}(\alpha, \beta) .
$$

Remark 1. It is of interest to note that $\mathcal{U C V}(\alpha, 0)=\mathcal{K}(\alpha)$ and $\mathcal{S}_{P}(\alpha, 0)=\mathcal{S}^{*}(\alpha)$.

Motivated by above definitions we define the following subclasses of $\mathcal{A}$ due to Murugusundaramoorthy and Magesh [16].

For $0 \leq \lambda<1, \quad 0 \leq \alpha<1$ and $\beta \geq 0$, we let $\mathcal{S}_{P}(\lambda, \alpha, \beta)$ be the subclass of $\mathcal{A}$ consisting of functions of the form (1) and satisfying the analytic criterion

$$
\begin{aligned}
& \Re\left(\frac{z f^{\prime}(z)}{(1-\lambda) f(z)+\lambda z f^{\prime}(z)}-\alpha\right) \\
& >\beta\left|\frac{z f^{\prime}(z)}{(1-\lambda) f(z)+\lambda z f^{\prime}(z)}-1\right|, z \in \mathbb{U},
\end{aligned}
$$

and also, let $\mathcal{U C V}(\lambda, \alpha, \beta)$ be the subclass of $\mathcal{A}$ consisting of functions of the form (1) and satisfying the analytic criterion

$\mathfrak{R}\left(\frac{f^{\prime}(z)+z f^{\prime \prime}(z)}{f^{\prime}(z)+\lambda z f^{\prime \prime}(z)}-\alpha\right)>\beta\left|\frac{f^{\prime}(z)+z f^{\prime \prime}(z)}{f^{\prime}(z)+\lambda z f^{\prime \prime}(z)}-1\right|, z \in \mathbb{U}$. (8)

We further let $\mathcal{T S}_{P}(\lambda, \alpha, \beta)=\mathcal{S}_{P}(\lambda, \alpha, \beta) \cap \mathcal{T}$ and $\mathcal{U C T}(\lambda, \alpha, \beta)=\mathcal{U C V}(\lambda, \alpha, \beta) \cap \mathcal{T}$.

Suitably specializing the parameters we note that

1. $\mathcal{T S}_{P}(0, \alpha, \beta)=\mathcal{T S}_{P}(\alpha, \beta)[6]$

2. $\mathcal{T S}_{P}(0,0, \beta)=\mathcal{T S}_{P}(\beta)[24]$

3. $\mathcal{T S}_{P}(0, \alpha, 1)=\mathcal{T S}_{P}(\alpha)[6]$

4. $\mathcal{T S}_{P}(\lambda, \alpha, 0)=\mathcal{T}^{*}(\lambda, \alpha)[1]$

5. $\mathcal{T S}_{P}(0, \alpha, 0)=\mathcal{T}^{*}(\alpha)[21]$

6. $\mathcal{U C T}(0, \alpha, \beta)=\mathcal{U C T}(\alpha, \beta)[6]$

7. $\mathcal{U C T}(0,0, \beta)=\mathcal{U C T}(\beta)[23]$

8. $\mathcal{U C T}(0, \alpha, 1)=\mathcal{U C T}(\alpha)[6]$

9. $\mathcal{U C T}(\lambda, \alpha, 0)=\mathcal{C}(\lambda, \alpha)[1]$

10. $\mathcal{U C T}(0, \alpha, 0)=\mathcal{C}(\alpha)$ [21].

Now we state the following characterization properties for the classes $\mathcal{S}_{P}(\lambda, \alpha, \beta), \mathcal{T S}_{P}(\lambda, \alpha, \beta), \mathcal{U C V}(\lambda, \alpha, \beta)$ and $\mathcal{U C T}(\lambda, \alpha, \beta)$ due to Murugusundaramoorthy and Magesh [16].
Theorem 1. A function $f(z)$ of the form (1) is in $\mathcal{S}_{P}(\lambda, \alpha, \beta)$ if

$$
\sum_{n=2}^{\infty} \backslash[n(1+\beta)-(\alpha+\beta)(1+n \lambda-\lambda)]\left|a_{n}\right| \leq 1-\alpha .
$$

Theorem 2. A function $f(z)$ of the form (2) is in $\mathcal{T S}_{P}(\lambda, \alpha, \beta)$ if and only if

$$
\begin{aligned}
& \sum_{n=2}^{\infty}\left\lceil[n(1+\beta)-(\alpha+\beta)(1+n \lambda-\lambda)]\left|a_{n}\right|\right. \\
& \leq 1-\alpha .
\end{aligned}
$$

Theorem 3. A function $f(z)$ of the form (1) is in $\mathcal{U C V}(\lambda, \alpha, \beta)$ if

$$
\begin{aligned}
& \sum_{n=2}^{\infty} n[n(1+\beta)-(\alpha+\beta)(1+n \lambda-\lambda)]\left|a_{n}\right| \\
& \leq 1-\alpha^{\dagger} .
\end{aligned}
$$

Theorem 4. A function $f(z)$ of the form (2) is in $\mathcal{U C T}(\lambda, \alpha, \beta)$ if and only if

$$
\begin{aligned}
& \sum_{n=2}^{\infty}\left\lceil n[n(1+\beta)-(\alpha+\beta)(1+n \lambda-\lambda)]\left|a_{n}\right|\right. \\
& \leq 1-\alpha .
\end{aligned}
$$

We recall here a generalized Bessel function $\omega(p, b, c)=\omega$ defined in [2] and given by

$$
\begin{aligned}
& \omega(z)=\omega(p, b, c) \\
& =\sum_{n=0}^{\infty} \frac{(-1)^{n} c^{n}}{n ! \Gamma\left(p+n+\frac{b+1}{2}\right)}\left(\frac{z}{2}\right)^{2 n+p} \uparrow
\end{aligned}
$$

which is the particular solution of the second order linear homogeneous differential equation

$$
z^{2} \omega^{\prime \prime}(z)+b z \omega^{\prime}(z)+\left[c z^{2}-p^{2}+(1-b)\right] \omega(z)=0
$$

where $b, p, c \in \mathbb{C}$, which is natural generalization of Bessel's equation. The differential equation (14) permits the study of Bessel function, modified Bessel function, spherical Bessel function and modified spherical Bessel functions all together. Solutions of (14) are referred to as the generalized Bessel function of order $\mathrm{p}$. The particular solution given by (13) is called the generalized Bessel function of the first kind of order p. Although the series defined above is convergent everywhere, the function $\omega_{p, b, c}$ is generally not univalent in $\mathbb{U}$. It is of interest to note that when $b=c=1$ we re-obtain the Bessel function of the first kind $\omega_{p, 1,1}=J_{p}$, and for $c=-1, b=1$ the function $\omega_{p, 1,-1}$ becomes the modified Bessel function $\mathcal{I}_{p}$ Now, we consider the function $u_{p, b, c}(z)$ defined by the transformation

$$
\begin{aligned}
& u_{p, b, c}(z)=2^{p} \Gamma\left(p+\frac{b+1}{2}\right) z^{\frac{-p}{2}} \omega_{p, b, c}(\sqrt{z}), \\
& \sqrt{1}=1 .
\end{aligned}
$$

By using well known Pochhammer symbol (or the shifted factorial) defined, in terms of the familiar Gamma function, by 


$$
\begin{aligned}
& (a)_{n}:=\frac{\Gamma(a+n)}{\Gamma(a)} \\
& = \begin{cases}1 & (n=0), \\
a(a+1)(a+2) \cdots(a+n-1) & (n \in \mathbb{N}:=\{1,2,3, \ldots\}) .\end{cases}
\end{aligned}
$$

we can express $u_{p, b, c}(z)$ as

$$
u_{p, b, c}(z)=\sum_{n=0}^{\infty} \frac{(-c / 4)^{n}}{\left(p+\frac{b+1}{2}\right)_{n}}\left(\frac{z^{n}}{n !}\right)
$$

where $\left(p+\frac{b+1}{2}\right) \neq 0,-1,-2, \ldots$. This function is analytic on $\mathbb{C}$ and satisfies the second-order linear differential equation

$$
4 z^{2} u^{\prime \prime}(z)+2(2 p+b+1) z u^{\prime}(z)+c z u(z)=0 .
$$

Now, we considered the linear operator

$$
\mathcal{I}(c, m): \mathcal{A} \rightarrow \mathcal{A}
$$

defined by

$$
\begin{aligned}
& \mathcal{I}(c, m) f(z)=z u_{p, b, c}(z)^{*} f(z) \\
& =z+\sum_{n=2}^{\infty} \frac{(-c / 4)^{n-1}}{(m)_{n-1}(n-1) !} a_{n} z^{n}
\end{aligned}
$$

where $m=p+\frac{(b+1)}{2} \neq 0$. For convenience throughout in the sequel, we use the following notations

$$
u_{p, b, c}=u_{p}, \quad m=p+\frac{b+1}{2} .
$$

and for if $c<0, m>0(m \neq 0,-1,-2, \ldots)$ let,

$$
z\left(2-u_{p}(z)\right)=z-\sum_{n=2}^{\infty} \frac{(-c / 4)^{n-1}}{(m)_{n-1}(n-1) !} z^{n_{\varkappa}} .
$$

The generalized Bessel function is a recent topic of study in Geometric Function Theory (e.g. see the work of $[2,3,4,5]$ and [14]. Motivated by results on connections between various subclasses of analytic univalent functions by using hypergeometric functions (see $[8,13,15,22,25]$ ) and by work of Baricz $[2,3,4,5]$, we obtain necessary and sufficient condition for the function $z\left(2-u_{p}(z)\right)$ to belong to the classes $\mathcal{T S}_{P}(\lambda, \alpha, \beta)$ and $\mathcal{U C T}(\lambda, \alpha, \beta)$

\section{Main Results and Their Consequences}

Lemma 1. [5] If $b, p, c \in \mathbb{C}$ and $m \neq 0,-1,-2, \ldots$ then the function $u_{p}$ satisfies the recursive relation

$$
4 m u_{p}^{\prime}(z)=-c u_{p+1}(z)
$$

for all $z \in \mathbb{C}$

Theorem 5. If $c<0$ and $m>0$ then $z\left(2-u_{p}(z)\right) \in \mathcal{T S}_{P}(\lambda, \alpha, \beta)$ if and only if $[1+\beta-\lambda(\alpha+\beta)] u_{p}^{\prime}(1)+(1-\alpha)\left[u_{p}(1)-1\right] \leq 2(1-\alpha)$.

Proof. Since

$$
z\left(2-u_{p}(z)\right)=z-\sum_{n=2}^{\infty} \frac{\left(\frac{-c}{4}\right)^{n-1}}{(m)_{n-1}(n-1) !} z^{n_{\uparrow}}
$$

according to Theorem 2, we must show that

$$
\begin{aligned}
& \sum_{n=2}^{\infty} \Uparrow[n(1+\beta)-(\alpha+\beta)(1+n \lambda-\lambda)] \frac{\left(\frac{-c}{4}\right)^{n-1}}{(m)_{n-1}(n-1) !}(20) \\
& \leq(1-\alpha)
\end{aligned}
$$

Now,

$\sum_{n=2}^{\infty}[n(1+\beta)-(\alpha+\beta)(1+n \lambda-\lambda)] \frac{\left(\frac{-c}{4}\right)^{n-1}}{(m)_{n-1}(n-1) !}$

$\leq \sum_{n=2}^{\infty} *\left[\begin{array}{l}(n-1)[1+\beta-\lambda(\alpha+\beta)] \\ +(1-\alpha)\end{array}\right] \frac{\left(\frac{-c}{4}\right)^{n-1}}{(m)_{n-1}(n-1) !}$

$=[1+\beta-\lambda(\alpha+\beta)] \sum_{n=1}^{\infty} \nmid n \frac{\left(\frac{-c}{4}\right)^{n}}{(m)_{n}(n) !}+(1-\alpha) \sum_{n=1}^{\infty} \nmid \frac{\left(\frac{-c}{4}\right)^{n}}{(m)_{n}(n) !}$

$=[1+\beta-\lambda(\alpha+\beta)] \sum_{n=1}^{\infty} * \frac{\left(\frac{-c}{4}\right)^{n}}{(m)_{n}(n-1) !}+(1-\alpha) \sum_{n=1}^{\infty} \uparrow \frac{\left(\frac{-c}{4}\right)^{n}}{(m)_{n}(n) !}$

$=\left[\begin{array}{l}1+\beta \\ -\lambda(\alpha+\beta)\end{array}\right] \sum_{n=1}^{\infty} * \frac{\left(\frac{-c}{4}\right)^{n+1}}{(m)_{n+1}(n) !}+(1-\alpha) \sum_{n=1}^{\infty} * \frac{\left(\frac{-c}{4}\right)^{n+1}}{(m)_{n+1}(n+1) !} !^{*}$

$=[1+\beta-\lambda(\alpha+\beta)] \frac{\left(\frac{-c}{4}\right)}{m} \sum_{n=1}^{\infty} *\left(\frac{\left(\frac{-c}{4}\right)^{n}}{(m+1)_{n}(n) !}\right)$

$+(1-\alpha) \sum_{n=1}^{\infty} \uparrow\left(\frac{\left(\frac{-c}{4}\right)^{n+1}}{(m)_{n+1}(n+1) !}\right)$

$=[1+\beta-\lambda(\alpha+\beta)] \frac{\left(\frac{-c}{4}\right)}{m} u_{p+1}(1)+(1-\alpha)\left[u_{p}(1)-1\right]$

$=[1+\beta-\lambda(\alpha+\beta)] u_{p}^{\prime}(1)+(1-\alpha)\left[u_{p}(1)-1\right]$.

But the last expression is bounded above by $1-\alpha$ if and only if (18) holds.

Remark 2. In particular when $c=-1$ and $b=1$, the condition 18 becomes

$$
2^{p-2} \Gamma(p+1)\left[\begin{array}{l}
{[1+\beta-\lambda(\alpha+\beta)] \mathcal{I}_{p+1}(1)} \\
+2(1-\alpha) \mathcal{I}_{p}(1)
\end{array}\right] \leq 1-\alpha,
$$

which is necessary and sufficient condition for $z\left(2-\zeta_{p}\left(z^{1 / 2}\right)\right)$ to be in $\mathcal{T S}_{P}(\lambda, \alpha, \beta)$ where

$$
\zeta_{p}\left(z^{1 / 2}\right)=2^{p} \Gamma(p+1) z^{-p / 2} \mathcal{I}_{p}\left(z^{1 / 2}\right)
$$

Theorem 6. If $c<0$ and $m>0$ then $z\left(2-u_{p}(z)\right)$ is in $\mathcal{U C T}(\lambda, \alpha, \beta)$ if and only if 


$$
\begin{aligned}
& {[1+\beta-\lambda(\alpha+\beta)] u_{p}^{\prime}(1)} \\
& +[3+2 \beta-\alpha-2 \lambda(\alpha+\beta)] u_{p}^{\prime}(1) \\
& +(1-\alpha) u_{p}(1) \leq 2(1-\alpha) .
\end{aligned}
$$

Proof. In view of Theorem 4,we need to show that

$$
\begin{aligned}
& \sum_{n=2}^{\infty} \llbracket[n(1+\beta)-(\alpha+\beta)(1+n \lambda-\lambda)] \frac{\left(\frac{-c}{4}\right)^{n-1}}{(m)_{n-1}(n-1) !} \\
& \leq(1-\alpha) .
\end{aligned}
$$

Now

$\sum_{n=2}^{\infty}\left\lceil[n(1+\beta)-(\alpha+\beta)(1+n \lambda-\lambda)] \frac{\left(\frac{-c}{4}\right)^{n-1}}{(m)_{n-1}(n-1) !}\right.$

$\leq \sum_{n=0}^{\infty} \uparrow(n+2)\left[\begin{array}{l}(n+2)(1+\beta) \\ -(1+\lambda(n+2)-\lambda)(\alpha+\beta)\end{array}\right] \frac{\left(\frac{-c}{4}\right)^{n+1}}{(m)_{n+1}(n+1) !}$

$\left.=[1+\beta-\lambda(\alpha+\beta)] \sum_{n=0}^{\infty}\right\rceil(n+2)^{2} \frac{\left(\frac{-c}{4}\right)^{n+1}}{(m)_{n+1}(n+1) !}$

$\left.-(\alpha+\beta)(1-\lambda) \sum_{n=0}^{\infty}\right\rceil(n+2) \frac{\left(\frac{-c}{4}\right)^{n+1}}{(m)_{n+1}(n+1) !}$

$\left\{\begin{array}{l}\sum_{n=0}^{\infty} \uparrow(n+1)^{2} \frac{\left(\frac{-c}{4}\right)^{n+1}}{(m)_{n+1}(n+1) !}+2 \sum_{n=0}^{\infty} \uparrow(n+1) \frac{\left(\frac{-c}{4}\right)^{n+1}}{(m)_{n+1}(n+1) !} \\ +\sum_{n=0}^{\infty} \frac{\left(\frac{-c}{4}\right)^{n+1}}{(m)_{n+1}(n+1) !}\end{array}\right\}$

$-(\alpha+\beta)(1-\lambda)\left\{\begin{array}{l}\sum_{n=0}^{\infty}(n+1) \frac{\left(\frac{-c}{4}\right)^{n+1}}{(m)_{n+1}(n+1) !} \\ +\sum_{n=0}^{\infty}{\frac{\left(\frac{-c}{4}\right)^{n+1}}{(m)_{n+1}(n+1) !}}^{*}\end{array}\right\}$

$=\left[\begin{array}{l}1+\beta \\ -\lambda(\alpha+\beta)\end{array}\right]\left\{\begin{array}{l}\sum_{n=0}^{\infty}(n+1) \frac{\left(\frac{-c}{4}\right)^{n+1}}{(m)_{n+1}(n) !} \\ +2 \sum_{n=0}^{\infty} \frac{\left(\frac{-c}{4}\right)^{n+1}}{(m)_{n+1}(n) !}+\sum_{n=0}^{\infty} \uparrow \frac{\left(\frac{-c}{4}\right)^{n+1}}{(m)_{n+1}(n+1) !}\end{array}\right\}$

$-(\alpha+\beta)(1-\lambda)\left\{\sum_{n=0}^{\infty} \frac{\left(\frac{-c}{4}\right)^{n+1}}{(m)_{n+1}(n) !}+\sum_{n=0}^{\infty} \frac{\left(\frac{-c}{4}\right)^{n+1}}{(m)_{n+1}(n+1) !}\right\}$

$=\left[\begin{array}{l}1+\beta \\ -\lambda(\alpha+\beta)\end{array}\right]\left\{\begin{array}{l}\sum_{n=0}^{\infty} \frac{\left(\frac{-c}{4}\right)^{n+1}}{(m)_{n+1}(n-1) !} \\ +3 \sum_{n=0}^{\infty} \frac{\left(\frac{-c}{4}\right)^{n+1}}{(m)_{n+1}(n) !}+\sum_{n=0}^{\infty} \nmid \frac{\left(\frac{-c}{4}\right)^{n+1}}{(m)_{n+1}(n+1) !}\end{array}\right\}$ $\left.-\left[\begin{array}{l}(\alpha+\beta) \\ (1-\lambda)\end{array}\right]\left\{\sum_{n=0}^{\infty} \frac{\left(\frac{-c}{4}\right)^{n+1}}{(m)_{n+1}(n) !}{ }^{*}+\sum_{n=0}^{\infty} \frac{\left(\frac{-c}{4}\right)^{n+1}}{(m)_{n+1}(n+1) !}\right\}\right\}$

$=\left.[1+\beta-\lambda(\alpha+\beta)] \sum_{n=0}^{\infty} \frac{\left(\frac{-c}{4}\right)^{n+1}}{(m)_{n+1}(n-1) !}\right|^{\dagger}$

$+[3+2 \beta-\alpha-2 \lambda(\alpha+\beta)] \sum_{n=0}^{\infty} \frac{\left(\frac{-c}{4}\right)^{n+1}}{(m)_{n+1}(n) !}{ }^{\dagger}$

$+(1-\alpha) \sum_{n=0}^{\infty} \frac{\left(\frac{-c}{4}\right)^{n+1}}{(m)_{n+1}(n+1) !}$

$=\left[\begin{array}{l}1+\beta \\ -\lambda(\alpha+\beta)\end{array}\right] \frac{\left(\frac{-c}{4}\right)^{2}}{m(m+1)} \sum_{n=0}^{\infty}\left(\frac{\left(\frac{-c}{4}\right)^{n-1}}{(m+2)_{n-1}(n-1) !}\right)$

$+\left[\begin{array}{c}3+2 \beta-\alpha \\ -2 \lambda(\alpha+\beta)\end{array}\right]^{\left(\frac{-c}{4}\right)} \frac{\left(\frac{-c}{4}\right)^{n}}{m} \sum_{n=0}^{\infty}\left(\frac{(m+1)_{n}(n) !}{(m)}\right.$

$+(1-\alpha) \sum_{n=0}^{\infty} \frac{\left(\frac{-c}{4}\right)^{n+1}}{(m)_{n+1}(n+1) !}$

$=[1+\beta-\lambda(\alpha+\beta)] \frac{\left(\frac{-c}{4}\right)^{2}}{m(m+1)} u_{p+2}(1)$

$+[3+2 \beta-\alpha-2 \lambda(\alpha+\beta)] \frac{\left(\frac{-c}{4}\right)}{m} u_{p+1}(1)$

$+(1-\alpha)\left[u_{p}(1)-1\right]$

$=[1+\beta-\lambda(\alpha+\beta)] u_{p}{ }_{p}(1)$

$+[3+2 \beta-\alpha-2 \lambda(\alpha+\beta)] u_{p}^{\prime}(1)$

$+(1-\alpha)\left[u_{p}(1)-1\right]$.

By a simplification, we see that the last expression is bounded above by $(1-\alpha)$ if and only if (22) holds.

\section{Inclusion Properties}

A function $f \in \mathcal{A}$ is said to be in the class $\mathcal{R}^{\tau}(A, B)$, $(\tau \in \mathbb{C} \backslash\{0\},-1 \leq B<A \leq 1)$, if it satisfies the inequality

$$
\left|\frac{f^{\prime}(z)-1}{(A-B) \tau-B\left[f^{\prime}(z)-1\right]}\right|<1 \quad(z \in \mathbb{U}) .
$$

The class $\mathcal{R}^{\tau}(A, B)$ was introduced earlier by Dixit and Pal [9]. If we put

$$
\tau=1, A=\beta \text { and } B=-\beta \quad(0<\beta \leq 1),
$$

we obtain the class of functions $f \in \mathcal{A}$ satisfying the inequality 


$$
\left|\frac{f^{\prime}(z)-1}{f^{\prime}(z)+1}\right|<\beta \quad(z \in \mathbb{U} ; 0<\beta \leq 1)
$$

which was studied by (among others) Padmanabhan [17] and Caplinger and Causey [7]. Making use of the following lemma, we will study the action of the Bessel function on the classes $\mathcal{U C T}(\lambda, \alpha, \beta)$

Lemma 2. [9] If $f \in \mathcal{R}^{\tau}(A, B)$ is of form (1), then

$$
\left|a_{n}\right| \leq(A-B) \frac{|\tau|}{n}, \quad n \in \mathbb{N} \backslash\{1\} .
$$

The result is sharp.

Theorem 7. Let If $c<0$ and $m>0$ If $f \in \mathcal{R}^{\tau}(A, B)$ and if the inequality

$$
(A-B)|\tau|\left\{\begin{array}{l}
{[1+\beta-\lambda(\alpha+\beta)] u_{p}^{\prime}(1)} \\
+(1-\alpha)\left[u_{p}(1)-1\right]
\end{array}\right\} \leq 1-\alpha
$$

is satisfied, then $\mathcal{I}(c, m)(f) \in \mathcal{U C T}(\lambda, \alpha, \beta)$.

Proof. Let $\mathrm{f}$ be of the form (1) belong to the class $\mathcal{R}^{\tau}(A, B)$ By virtue of Theorem 4, it suffices to show that $L(\alpha, \beta, \lambda)$

$$
=\sum_{n=2}^{\infty} \times\left[\begin{array}{l}
n(1+\beta) \\
-(\alpha+\beta)(1+n \lambda-\lambda)
\end{array}\right] \frac{\left(\frac{-c}{4}\right)^{n-1}}{(m)_{n-1}(n-1) !}\left|a_{n}\right| \leq 1-\alpha .
$$

Since $f \in \mathcal{R}^{\tau}(A, B)$ then by Lemma 2 we have $\left|a_{n}\right| \leq(A-B) \frac{|\tau|}{n}$. Hence

$L(\alpha, \beta, \lambda)=\sum_{n=2}^{\infty} n[n(1+\beta)-(\alpha+\beta)(1+n \lambda-\lambda)]$

$\frac{\left(\frac{-c}{4}\right)^{n-1}}{(m)_{n-1}(n-1) !}(A-B) \frac{|\tau|}{n}$

$\leq(A-B)|\tau|\left[\begin{array}{l}\sum_{n=2}^{\infty}\left[\begin{array}{l}n(1+\beta) \\ -(\alpha+\beta)(1+n \lambda-\lambda)\end{array}\right] \\ \frac{\left(\frac{-c}{4}\right)^{n-1}}{(m)_{n-1}(n-1) !}\end{array}\right]$

$\leq(A-B)|\tau|\left[\begin{array}{l}\sum_{n=2}^{\infty}\left[\begin{array}{l}(n-1)[1+\beta-\lambda(\alpha+\beta)] \\ +(1-\alpha)\end{array}\right] \\ \frac{\left(\frac{-c}{4}\right)^{n-1}}{(m)_{n-1}(n-1) !}\end{array}\right]$

$\leq(A-B)|\tau|\left[\begin{array}{l}(1+\beta-\lambda(\alpha+\beta)) \sum_{n=1}^{\infty} n{\frac{\left(\frac{-c}{4}\right)^{n}}{(m)_{n}(n) !}}^{\dagger} \\ +(1-\alpha) \sum_{n=1}^{\infty}{\frac{\left(\frac{-c}{4}\right)^{n}}{(m)_{n}(n) !}}^{\dagger}\end{array}\right]$

Further, proceeding as in Theorem 5

$$
\begin{aligned}
& L(\alpha, \beta, \lambda) \\
& \leq(A-B)|\tau|\left\{\begin{array}{l}
{[1+\beta-\lambda(\alpha+\beta)] \sum_{n=0}^{\infty}{\frac{\left(\frac{-c}{4}\right)^{n+1}}{(m)_{n+1}(n) !}}^{*}} \\
+(1-\alpha) \sum_{n=0}^{\infty}{\frac{\left(\frac{-c}{4}\right)^{n+1}}{(m)_{n+1}(n+1) !}}^{*}
\end{array}\right\} \\
& \leq(A-B)|\tau|\left\{\begin{array}{l}
{[1+\beta-\lambda(\alpha+\beta)] \frac{\left(\frac{-c}{4}\right)}{m} u_{p+1}(1)} \\
+(1-\alpha)\left[u_{p}(1)-1\right]
\end{array}\right\} \\
& \leq(A-B)|\tau|\left\{\begin{array}{l}
{[1+\beta-\lambda(\alpha+\beta)] u_{p}^{\prime}(1)} \\
+(1-\alpha)\left[u_{p}(1)-1\right]
\end{array}\right\}
\end{aligned}
$$

But this last expression is bounded above by $1-\alpha$ if and only if (23) holds.

Theorem 8. Let $c<0$ and $m>0$ then

$$
\mathcal{L}(m, c, z)=\int_{0}^{z} x\left(2-u_{p}(t)\right) d t
$$

is in $\mathcal{U C T}(\lambda, \alpha, \beta)$ if and only if inequality

$$
[1+\beta-\lambda(\alpha+\beta)] u_{p}^{\prime}(1)+(1-\alpha)\left[u_{p}(1)-1\right] \leq 1-\alpha .
$$

\section{Proof. Since}

$$
\mathcal{L}(m, c, z)=z-\sum_{n=2}^{\infty} \frac{\left(\frac{-c}{4}\right)^{n-1}}{(m)_{n-1}} \frac{z^{n}}{(n) !} !^{\dagger}
$$

then by Theorem 4 we need only to show that

$P(\alpha, \beta, \lambda)=\sum_{n=2}^{\infty} \mathrm{n}\left[\begin{array}{l}n(1+\beta) \\ -(\alpha+\beta)(1+n \lambda-\lambda)\end{array}\right]\left(\frac{\left(\frac{-c}{4}\right)^{n-1}}{(m)_{n-1} \mathrm{n} !}\right) *$

Now

$$
\begin{aligned}
& \sum_{n=2}^{\infty} \mathrm{n}[n(1+\beta)-(\alpha+\beta)(1+n \lambda-\lambda)]\left(\frac{\left(\frac{-c}{4}\right)^{n-1}}{(m)_{n-1} \mathrm{n} !}\right)^{x} \\
& \leq \sum_{n=2}^{\infty}[n(1+\beta)-(\alpha+\beta)(1+n \lambda-\lambda)]\left(\frac{\left(\frac{-c}{4}\right)^{n-1}}{(m)_{n-1}(n-1) !}\right)
\end{aligned}
$$

Further, proceeding as in Theorem 5

$P(\alpha, \beta, \lambda)$

$$
\begin{aligned}
& \leq\left[\begin{array}{l}
1+\beta \\
-\lambda(\alpha+\beta)
\end{array}\right] \sum_{n=0}^{\infty} \frac{\left(\frac{-c}{4}\right)^{n+1}}{(m)_{n+1}(n) !}+(1-\alpha) \sum_{n=0}^{\infty} \frac{\left(\frac{-c}{4}\right)^{n+1}}{(m)_{n+1}(n+1) !}{ }^{*} \\
& =[1+\beta-\lambda(\alpha+\beta)] \frac{\left(\frac{-c}{4}\right)}{m} u_{p+1}(1)+(1-\alpha)\left[u_{p}(1)-1\right] . \\
& =[1+\beta-\lambda(\alpha+\beta)] u_{p}^{\prime}(1)+(1-\alpha)\left[u_{p}(1)-1\right]
\end{aligned}
$$

which is bounded above by $1-\alpha$ if and only if (26) holds. 


\section{Concluding Remarks}

If we put $c=-1$ and $b=1$ in above theorems we obtain analogous results of (21). Further by taking $\beta=0$ and specializing the parameter $\lambda$ we can state various interesting results (as proved in above theorems) for the various subclasses listed in the introduction.

\section{Acknowledgement}

The authors thank the referees for their valuable suggestions to improve the paper in present form.

\section{References}

[1] O.Altintas and S.Owa, On subclasses of univalent functions with negative coefficients, Pusan Kyŏngnam Math.J., 4 (1988), 41-56.

[2] A. Baricz, Geometric properties of generalized Bessel functions, Publ. Math. Debrecen, 73 (1-2) (2008), 155-178.

[3] A. Baricz, Geometric properties of generalized Bessel functions of complex order, Mathematica, 48 (71) (1) (2006), 13-18.

[4] A. Baricz,Generalized Bessel functions of the first kind, $\mathrm{PhD}$ thesis, Babes-Bolyai University, Cluj-Napoca, (2008).

[5] A. Baricz,Generalized Bessel functions of the first kind, Lecture Notes in Math., Vol. 1994, Springer-Verlag (2010).

[6] R.Bharati, R.Parvatham and A.Swaminathan, On subclasses of uniformly convex functions and corresponding class of starlike functions, Tamkang J.Math., 6 (1) (1997), 17-32.

[7] T. R. Caplinger and W. M. Causey, A class of univalent functions, Proc. Amer. Math. Soc. 39 (1973), 357-361.

[8] N.E. Cho, S.Y.Woo and S. Owa, Uniform convexity properties for hypergeometric functions, Fract. Cal. Appl. Anal., 5 (3) (2002), 303-313.

[9] K. K. Dixit and S. K. Pal, On a class of univalent functions related to complex order, Indian J. Pure Appl. Math. 26 (9) (1995) 889896.
[10] A.W.Goodman, On uniformly convex functions, Ann.polon.Math., $56,(1991), 87-92$

[11] A.W.Goodman, On uniformly starlike functions, J.Math.Anal.Appl., 155, (1991), 364-370.

[12] S.Kanas and A. Wi'sniowska, Conic regions and k-uniform convexity, J. Comput. Appl. Math., 105 (1999), 327-336.

[13] E. Merkes and B.T. Scott, Starlike hypergeometric functions, Proc. Amer. Math. Soc., 12 (1961), 885-888.

[14] S.R. Mondal and A. Swaminathan, Geometric properties of Generalized Bessel functions, Bull. Malays. Math. Sci. Soc., 35 (1) (2012), 179-194.

[15] A.O.Mostafa, A study on starlike and convex properties for hypergeometric functions, Journal of Inequalities in Pure and Applied Mathematics., 10 (3), Art.87 (2009), 1-8.

[16] G.Murugusundaramoorthy and N.Magesh, On certain subclasses of analytic functions associated with hypergeometric functions, Appl. Math. Letters 24, (2011), 494-500.

[17] K. S. Padmanabhan, On a certain class of functions whose derivatives have a positive real part in the unit disc, Ann. Polon. Math. 23 (1970), 73-81.

[18] S. Ponnusamy and F. Rønning, Duality for Hadamard products applied to certain integral transforms, Complex Variables Theory Appl. 32 (1997), 263-287.

[19] F.Rønning, Uniformly convex functions and a corresponding class of starlike functions, Proc.Amer.Math.Soc., 118, (1993), 189-196.

[20] F.Rønning, Integral representations for bounded starlike functions, Annal.Polon.Math., 60, (1995), 289-297.

[21] H.Silverman, Univalent functions with negative coefficients, Proc.Amer.Math.Soc., 51 (1975), 109-116.

[22] H.Silverman, Starlike and convexity properties for hypergeometric functions, J.Math.Anal.Appl., 172 (1993), 574-581.

[23] K.G.Subramanian, G.Murugusundaramoorthy, P.Balasubrahmanyam and H.Silverman, Subclasses of uniformly convex and uniformly starlike functions. Math. Japonica, 42 (3), (1995), 517-522.

[24] K.G.Subramanian, T.V.Sudharsan, P.Balasubrahmanyam and H.Silverman, Classes of uniformly starlike functions, Publ. Math. Debrecen., 53 (3-4), (1998), 309-315.

[25] A.Swaminathan, Certain suffienct conditions on Gaussian hypergeometric functions, Journal of Inequalities in Pure and Applied Mathematics., 5 (4), Art. 83 (2004), 1-10. 